\title{
Fteknokultura
}

\#Ruiz, J.M. (2016). El rol del medialab en la Educación Artística Superior pública ecuatoriana: primeras experiencias en arte, ciencia y tecnología, Revista Teknokultura Vol. 13(1), 97-116.

Recibido: $01-02-2016$

Aceptado: 14-04-2016
Open peer review

http://revistas.ucm.es/index.php/TEKN/pages/view/opr-51773

\section{El rol del medialab \\ en la Educación Artística Superior pública ecuatoriana: primeras experiencias en arte, ciencia y tecnología}

\author{
The role of medialabs \\ in Ecuadorian public arts Higher Education: \\ first experiences in art, science and technology \\ José M. Ruiz \\ Universidad de las Américas \\ contacto@josemanuelruiz.net
}

\section{Resumen}

La educación artística universitaria pública en el Ecuador adolece de materias ligadas al estudio del espacio convergente actual entre arte, ciencia y tecnología y sus respectivas prácticas creativas. Ante esta situación, que denota cierto anquilosamiento bajo técnicas y perfiles tradicionales, son los nuevos medialabs creados en los últimos años en el contexto de las Facultades de Arte de la Universidad de Cuenca y de la Universidad Central del Ecuador (Quito), los que vienen implementando las primeras prácticas en este sentido, cu- 
briendo así las carencias curriculares de dichas carreras en lo que a cultura digital, arte y nuevos medios se refiere. Este estudio analiza las características de estos centros y la metodología seguida para introducir el arte y las nuevas tecnologías de forma pionera en el país.

\section{Palabras Clave}

medialab; educación superior; Ecuador; arte; ciencia; tecnología.

\section{Abstract}

Public university art education in Ecuador lacks subjects to study the current convergent space between art, science and technology and their creative practices. This situation reveals a certain stagnation under traditional techniques and profiles. The new medialabs of the Faculty of Arts (University of Cuenca and Central University) from Ecuador (Quito) are implementing the first practices in this regard, repairing the curriculum deficiencies of these career paths in digital culture and new media art. This study analyzes the characteristics of these centers and the methodology followed to introduce the art and new technologies pioneered in the country.

\section{KEYWORDS}

Higher Education; Ecuador; art; science; technology. 


\section{Contenidos}

1. Introducción

2. El medialab como nodo experiencial actual en arte, ciencia y tecnología

3. Metodología

4. Mallas curriculares y perfil de egresados en la Educación Artística Superior pública ecuatoriana

5. Estudio de casos: los medialabs de la Universidad de Cuenca y Universidad Central

6. Conclusiones

7. Referencias

\section{Contents}

1. Introduction

2. Medialab like an actual experiential node in art, science and technology.

3. Methodology.

4. Curricula and graduate profiles in Ecuadorian public art Higher Education.

5. Case study: medialabs of the Cuenca University and Central University.

6. Conclusions

7. References 



\section{Introducción}

Durante años, el arte producido en Ecuador se ha mantenido ligado principalmente al uso de las técnicas tradicionales, las cuales siguen teniendo un enorme peso y espacio en su contexto cultural. Obras de artistas de la talla de Guayasamín, vinculadas estéticamente a los movimientos de vanguardia de la primera mitad del siglo XX, han dotado al país de un fuerte legado iconográfico. Su arte caló sobremanera en el imaginario colectivo de la población. Es por ello que, el espacio concreto para un arte del siglo 21 -que en países latinoamericanos como Colombia, Chile o Brasil, entre otros, es más fuerte y está mejor visibilizado-, en el país ecuatoriano es reducido y extremadamente minoritario. Sin embargo, gracias a los aportes de extrema valía de artistas, movimientos, organizaciones y, en última instancia, de las universidades públicas -como estudiaremos en el transcurso de la presente investigación- se comienza a atisbar un ligero viraje en los intereses procesales de las nuevas generaciones.

Deberíamos entender las técnicas y las tecnologías empleadas en las artes como dinámica creativa antes que simples estructura repetitivas, y huir del rechazo de las técnicas como meros procedimientos instrumentales, afirmando los procesos operativos como campo de investigación autosuficiente y, según como, final (Alsina, 2007, p. 24).

Es así como, desde el año 2012, fecha en la que se inauguró el medialab de la Universidad de Cuenca -el primer medialab fomentado desde la universidad pública ecuatoriana-, que se vienen dando una serie de acontecimientos y circunstancias ligadas al ámbito creativo-educacional dignas de estudio en el país latinoamericano. Igualmente, en 2014 se comenzó a engendrar la idea de la creación de un nuevo medialab, en este caso adscrito a la Facultad de Artes de la Universidad Central del Ecuador (UCE), en Quito. Este proyecto, no sin dificultades importantes -largos procesos burocráticos, extensos debates, propuestas y adaptaciones por parte de un buen número de investigadores nacionales e internacionales que participaron de forma activa en el proceso-, finalmente concluyó con la aprobación del Rector de la Universidad Central a principios del 2016.

En este preciso momento, se está pensando en las posibilidades de implementación de un medialab en la extensa Universidad de las Artes, ubicada en Guayaquil, hecho que, de alcanzarse el objetivo y llevar a buen puerto la propuesta, correspondería al tercer medialab vinculado a la universidad pública de Ecuador. Un hecho sin precedentes en América La- 
tina. Esto proporciona una evidencia que lleva a afirmar que parte de la educación en el país - la más ligada a las prácticas artísticas y a la creatividad- se encuentra en plena transformación y adecuación a las nuevas necesidades de la contemporaneidad actual. Pero, ¿qué características posee un medialab para despertar tanto interés en los mayores representantes de la universidad pública de Ecuador? ¿Qué prácticas se desarrollan en su contexto? ¿Cuál es el rol específico del medialab en la universidad pública ecuatoriana? ¿Y cuáles sus aportaciones más relevantes a esta?

El presente estudio fija su atención de forma particular en las condiciones, características y metodología de estos nuevos centros o laboratorios, analizando los modelos o referentes seguidos por los mismos y la presencia e importancia que poseen sobre las entidades a las cuales se adscriben, en este caso, las universidades públicas ecuatorianas.

\section{EI medialab como nodo experiencial actual en arte, ciencia y tecnología}

Desde que en 1963 el físico especialista en láser Billy Klüver estableciera relaciones interdisciplinares con artistas, científicos e ingenieros en el contexto de E.A.T. ${ }^{1}$ (Experiments in Art and Technology) en Nueva York, a lo que le siguió en 1967 la creación del C.A.V.S. (Center for Advanced Visual Studies), integrado en el Massachusetts Institute of Technology y fundado por György Kepes o, unos años más tarde, el programa The Generative Systems impulsado por la artista Sonia L. Sheridan en el Art Institute de Chicago, muchos han sido los centros fundados en torno a la relación experiencial entre arte, ciencia y tecnología. En esta línea, se forma un largo bagaje de hitos históricos y artísticos internacionales mediante una apuesta por la producción creativa de vanguardia interdisciplinar con nuevos medios, los cuales fueron agrupados bajo el término genérico "medialab", como expuso Ruiz Martín (2014) en su tesis doctoral Aparición, impacto y efectos de la máquina automática en el atelier del artista. Del taller tradicional al medialab.

Para la presente investigación resulta imprescindible, definir lo más adecuadamente posible el término principal que nos trata: medialab. Si nos referimos al significado etimológico de la palabra, caeremos en la cuenta de que se trata de un anglicismo, pues 'medialab' sería una unión entre dos conceptos diferenciados y a la vez enraizados: medios y

\footnotetext{
${ }^{1}$ Fred Waldhauer, Billy Klüver, Robert Rauschenberg y Robert Whitman fundaron esta agrupación interdisciplinar con el objetivo de promover la colaboración entre artistas, científicos e ingenieros, basándose en el diálogo, la investigación y la producción de obras experimentales que convergían en la relación entre arte, ciencia y tecnología.
} 
laboratorio o, expuesto de otro modo, laboratorio de medios. Es evidente que para que exista laboratorio debe de producirse investigación o experimentación, pues la Real Academia Española de la Lengua lo define como sigue: "Lugar dotado de los medios necesarios para realizar investigaciones, experimentos y trabajos de carácter científico o técnico".

Por tratarse en cierta medida de un término reciente y con la intención de otorgarle un contexto de máxima actualidad, resulta imprescindible la referencia al contexto de la Revolución Tecnológica o cultura digital. Y ¿qué define tal revolución? La revolución en los lenguajes, la revolución en los dispositivos y la revolución en la red. Así, se propone la siguiente como definición: "Espacio físico-virtual dotado de los nuevos medios y dispositivos tecnológicos necesarios para realizar investigaciones, experimentos y trabajos de carácter interdisciplinar" (Ruiz, 2014, p. 202). A este respecto, Alcalá y Maisons (1993) proponen una definición que vierte luces en su Estudio/Propuesta para la creación de un Centro de Excelencia en Arte y Nuevas Tecnologías, realizado por encargo de la Fundación Telefónica: "El medialab como la nueva basílica de la organización de los discursos, como lugar de encuentro del viajero y escenario de todas las experiencias colectivas requiere del sometimiento individual a las formulaciones de sus nuevas reglas de juego" (Alcalá, 1993, p. $8)$.

Pero el medialab supone mucho más, ya que representa un ejemplo de elevado interés para perpetrar el obligado estudio sobre el cambio de modelo educativo y cultural -que se inicia con el fin de los mass media-, en el que el consumidor -o alumno o colaborador- se transforma de un ser pasivo a un ser activo, convirtiendo el acto comunicativo en un feedback constante de información. Un cambio paradigmático -ya analizado por diversos expertos en la materia, entre los que destaca el abogado y académico Lawrence Lessig (2005a, 2005b, 2008)- protagonizado por un relevo generacional poseedor de los nuevos medios y cohabitante de nuevas plataformas de carácter virtual.

Las artes, entendidas ya no solo como creadoras de objetos exhibitivos sino también como generadoras de comportamientos, de situaciones a partir de las cuales es posible generar nuevas formas de relacionarse con el mundo y la realidad, buscan también establecer esos espacios de comunicación entre hombre, máquina y sociedad generando nuevas inter faces como elementos de conexión que facilitan el intercambio de datos (Alsina, 2007, p. 29).

Con todo ello, el medialab actual -que pasa de ser un espacio de creación interdisciplinar donde el artista lideraba el grupo, a generar diálogos transdisciplinares de enorme interés- 
cifra sus objetivos en la redefinición de diversos conceptos ligados con la educación, la cultura y la comunicación:

Contribuye a la construcción de una nueva metáfora del mundo, que ya no es, una vez más, un sistema conocido y consolidado, sino que ha vuelto a mostrarse como un nuevo sistema, una nueva entidad que ha trastocado toda nuestra relación hacia él y hacia los sis temas de conocimiento y evaluación de sus fenómenos (Alcalá \& Maisons, 2004, p. 8).

De lo que no cabe duda $-\mathrm{y}$ esto es algo genérico que puede aplicarse a todos los centros actuales-, es que comparten el uso de tecnologías digitales como medios procesuales, alimentando interacciones de gran interés entre arte, ciencia y tecnología, como lo hicieran desde la década de los 60 los protagonistas y colaboradores de los centros anteriormente mencionados y otros posteriores como ZKM, ARS Electronica, NTT InterCommunication Center o V2, por citar algunos de ellos.

\section{Metodología}

Con el fin de determinar qué papel o rol está jugando el medialab en la universidad pública de Ecuador y analizar las características y modelo implementado, se ha empleado una metodología de investigación cualitativa -principalmente descriptiva y comparativa-, donde el estudio de casos y la observación de los datos documentales puestos al servicio del investigador a través de las diferentes plataformas, tanto físicas como virtuales, han generado el corpus fundamental de la misma. En este sentido, cabe señalar que el investigador que suscribe ha participado como experto de forma directa del proceso de elaboración del proyecto UCE Lab (en colaboración activa con los investigadores Christian Viteri, Javier Escudero, Arantxa Masachs y Susan Rocha, entre otros) para el desarrollo de los objetivos, líneas estratégicas y laboratorios del centro, por lo que su situación y experiencia específica le ha proporcionado información y materiales inéditos que merecen ser publicados para el conocimiento colectivo internacional y su respectiva y posterior discusión.

En primer lugar, se levantaron los datos de las mallas curriculares de las principales carreras (correspondientes a universidades públicas de Ecuador) implicadas en los citados medialabs, esto es, las carreras de artes plásticas-artes visuales, tanto de la Universidad Central como de la Universidad de Cuenca, realizando un análisis comparativo de las mismas. Se empleó para ello una serie de indicadores que responden a campos de estudio 
muy específicos y donde pueden incluirse la mayor parte de materias impartidas en sus pregrados:
a) Técnicas tradicionales (véase dibujo, pintura, escultura, etc.)
b) Historia, teoría y estudios del arte
c) Gestión cultural
d) Investigación
e) Nuevas tecnologías
f) Otros

Posteriormente, se analizaron minuciosamente los objetivos y líneas estratégicas que forman parte de los programas base de los dos medialabs. Se estudiaron detenidamente ambos casos y se clasificó dicha información según los indicadores anteriormente citados, con el fin de dotar de mayor validez y rigor a la práctica comparativa señalada.

Por último, la confrontación de todos los datos recabados -dispuestos en tablas y gráficos que facilitan la propia interpretación del lector- ha permitido la extracción de las conclusiones respectivas.

Resulta imprescindible señalar que la presente investigación parte de una anterior que culminó con la defensa y aprobación de la Tesis Doctoral Aparición, impacto y efectos de la máquina automática en el atelier del artista. Del taller tradicional al medialab ${ }^{2}$. En ella se analizaron -entre otras cuestiones- los diferentes modelos de medialabs a nivel nacional Arteleku (San Sebastián), MIDE (Cuenca), Hangar (Barcelona), MECAD (Sabadell), LABoral (Gijón), Medialab-Prado (Madrid), CCBLAB (Barcelona) y eTOPIA (Zaragoza), e internacional -E.A.T. (New York), C.A.V.S. (Massachusetts), The Generative Systems (Chicago), Constant Variable (Bruselas), MIT Medialab (Massachusetts), V2_ (Rotterdam), ZKM (Karlsruhe), Centro Multimedia (México D.F.), ARS Electronica Center (Linz) y NTT ICC (Tokio), visitando los estatales en su totalidad y dos paradigmáticos y con realidades muy diferenciadas a nivel internacional, ZKM y Constant Variable. Este hecho ha permitido localizar los modelos o referentes seguidos por los centros que nos competen en la presente investigación.

\footnotetext{
${ }^{2}$ Tesis Doctoral de José Manuel Ruiz, dirigida por José Ramón Alcalá y defendida el 4 de marzo de 2014 en el Departamento de Arte de la Facultad de Bellas Artes de Cuenca - Universidad de Castilla-La Mancha, que obtuvo la calificación de Sobresaliente Cum Laude el 19 de marzo de 2014.
} 


\section{Mallas curriculares y perfil de egresados en la Educación Artística Superior pública ecuatoriana}

Tabla 1: Materias impartidas por ejes en Grados de Artes Plásticas/Visuales, Universidad Central y Universidad de Cuenca

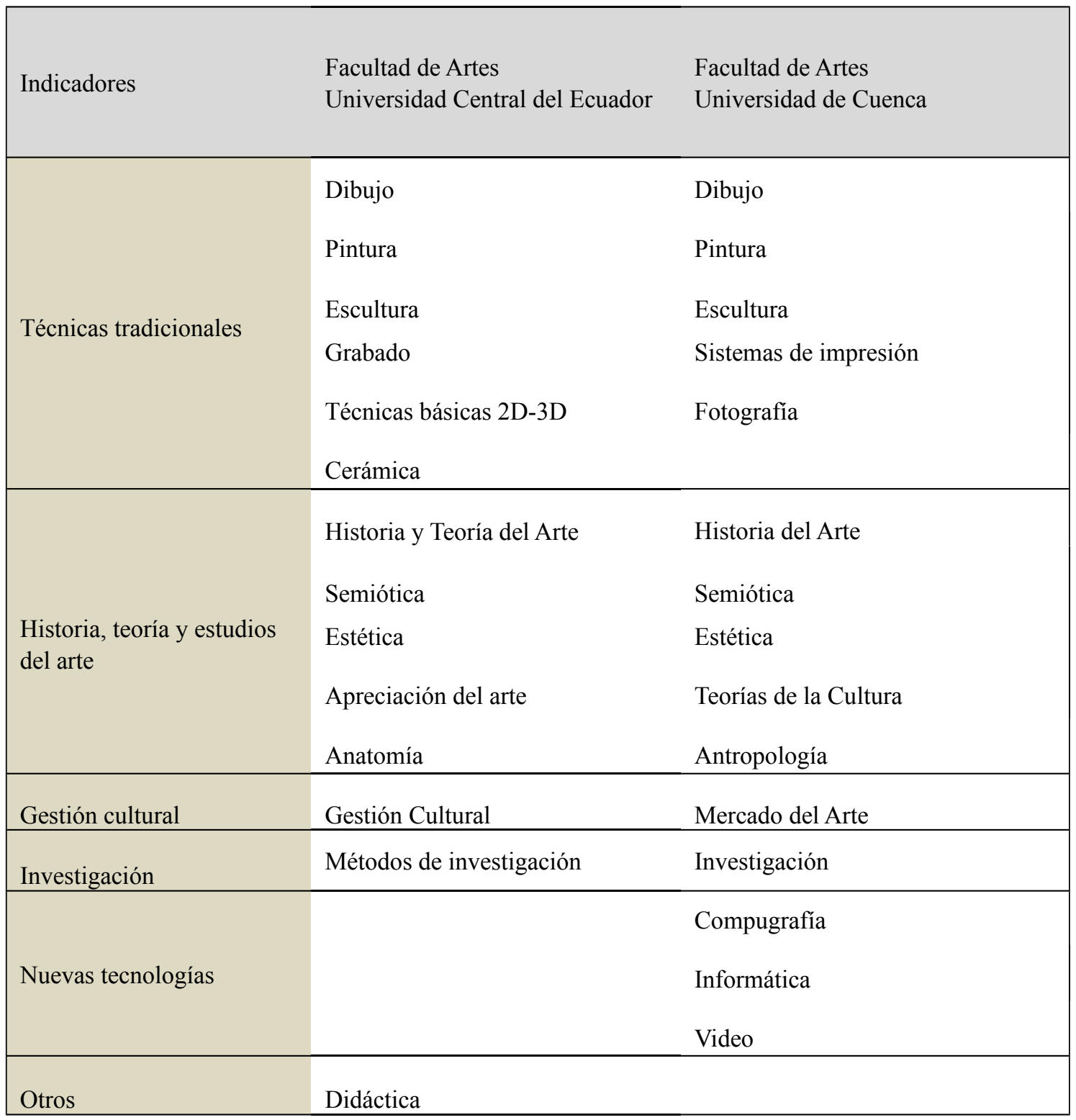

Fuente: Elaboración propia basándonos en los materiales recabados. 
En la actualidad, las mallas curriculares de las carreras de artes visuales en la Educación Superior artística de carácter pública en Ecuador sufren de un estancamiento notable. Este anquilosamiento, ejemplificado en la enseñanza de las técnicas tradicionales ${ }^{3}$ desde una visión eminentemente academicista, requiere de una actualización por parte de las autoridades competentes. Esta actualización inmediata o progresiva debe encaminarse hacia la adaptación de las nuevas necesidades y arengas de la cultura digital, pues con la digitalización y la introducción de la telemática en la vida cotidiana del ser humano, tanto el arte como la ciencia y la tecnología se sitúan en un contexto de mayor complejidad que demanda una participación activa de la comunidad artística mediante el análisis, el estudio y la práctica.

Tal y como se puede observar en las siguientes tablas y gráficos ${ }^{4}$ (Tabla 1, Gráfico 1 y Gráfico 2), las mallas curriculares de las dos carreras estudiadas comparten una buena parte de materias que, en este caso, han servido de indicadores para establecer un diagnóstico sobre los perfiles de las mismas. Es reseñable que se mantienen fieles, en términos generales, a la enseñanza de las técnicas tradicionales y a la impartición de materias que ahondan en aspectos históricos y teóricos, como 'Estética', 'Semiótica' e 'Historia del Arte', aunque sin penetrar en los cambios de paradigma más actuales. Además, comparten igualmente la inclusión de una materia relacionada con la investigación.

Si bien, en el caso de la Facultad de Artes Visuales de la Universidad de Cuenca y a diferencia de la Facultad de Artes Plásticas de la Universidad Central, se observa una apertura hacia nuevas vías y prácticas artísticas, más ligadas a la contemporaneidad y los nuevos medios, como en el caso específico de las asignaturas de 'Compugrafía', 'Informática' y 'Video', lo que nos arroja un dato de extrema importancia, algo que puede visualizarse mejor en los siguientes gráficos:

\footnotetext{
${ }^{3}$ Véanse dibujo, pintura, escultura, grabado, cerámica, etc.

${ }^{4}$ Cada una de las tablas y gráficos presentes en el texto fueron realizadas por el autor a partir de materiales recabados en la investigación.
} 
Gráfico 1: Materias impartidas por ejes en Grados de Artes Plásticas/Visuales, Universidad Central

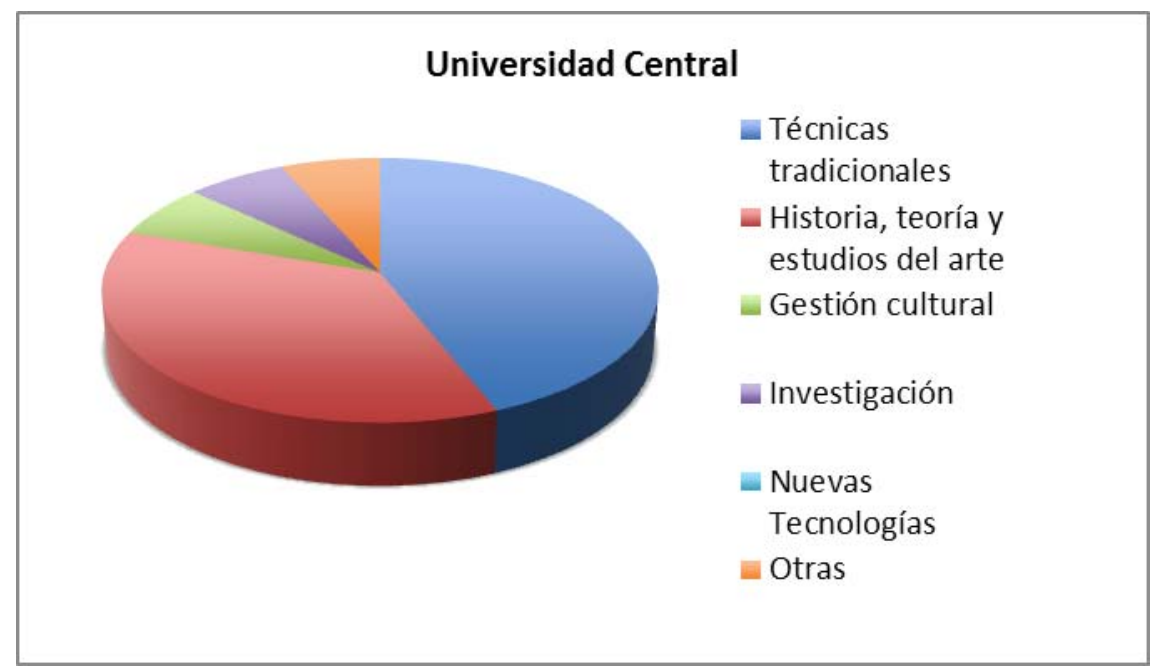

Fuente: Elaboración propia basándonos en los materiales recabados.

Gráfico 2: Materias impartidas por ejes en Grados de Artes Plásticas/Visuales, Universidad de Cuenca

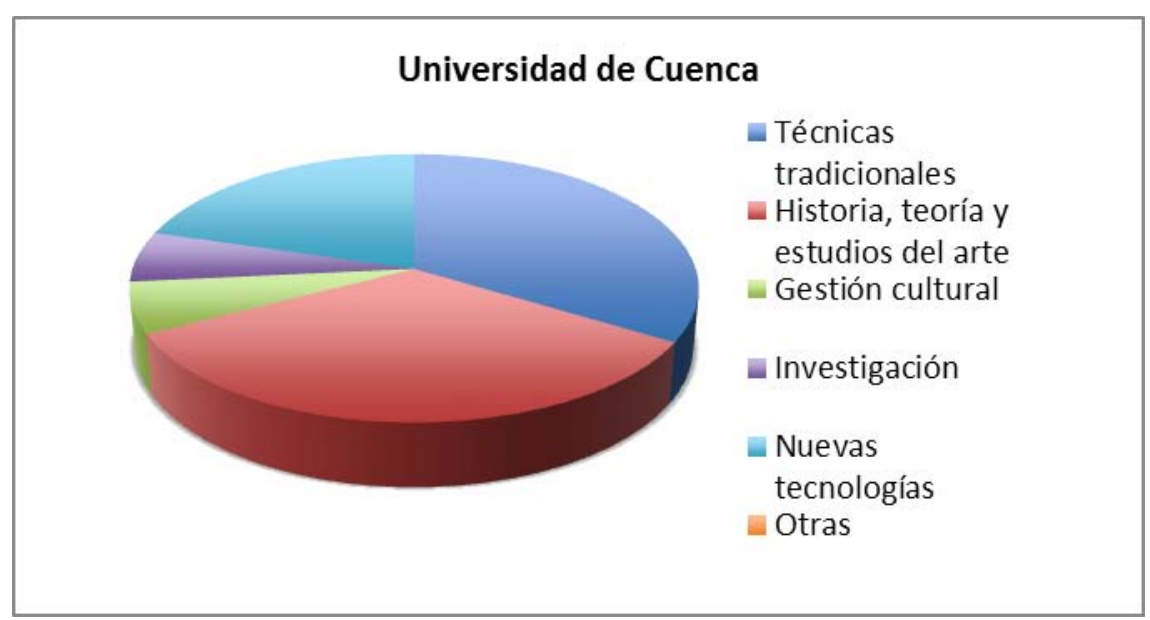

Fuente: Elaboración propia basándonos en los materiales recabados.

Así pues, el análisis de las materias pertenecientes a las mallas curriculares de estas dos facultades establece ligeras diferencias entre los perfiles de egresados, evidenciadas 
principalmente por la implementación de materias ligadas a las nuevas tecnologías en la Facultad de Artes de la Universidad de Cuenca, a diferencia del caso de la misma Facultad de la Universidad Central en Quito.

\section{Estudio de casos:}

\section{los medialabs de la Universidad de Cuenca y Universidad Central}

Desde 2012, año de su fundación, el MediaLab de la Universidad de Cuenca, estableciendo su espacio físico en uno de los laboratorios de su Facultad de Artes, viene desarrollando una labor de gran interés e innovación en el contexto ecuatoriano. Como bien se indica en su website:

La Facultad de Artes de la Universidad de Cuenca es una de las pocas instituciones que oferta programas académicos de educación superior en Arte y que en los últimos años ha mantenido un interés en los procesos tecnológicos y su integración con la práctica artística y el diseño (Medialabcuenca.org, 2016).

Entre sus objetivos se encuentran los siguientes:

- Promover la investigación, creación y desarrollo de las artes, el diseño y la tecnología con una visión interdisciplinaria, potenciando el trabajo colaborativo de estudiantes, docentes e investigadores de la Facultad de Artes y la Universidad de Cuenca.

- Formalizar al MediaLab como el centro tecnológico de la Facultad de Artes, un espacio de gestión de recursos materiales, teóricos y de know how, que integra diversas cátedras y proyectos de la Facultad.

- Impulsar proyectos artísticos y de diseño con medios tecnológicos en diferentes soportes, técnicas y medios para promover el intercambio multidisciplinario de conocimientos a nivel local, regional e internacional.

- Desarrollar proyectos con la participación de estudiantes y docentes con diferentes niveles de aplicación tecnológicos y con gran impacto en la sociedad.

- Vincular proyectos de investigación en las áreas de las artes, diseño y tecnología con la comunidad, con el fin de aportar a la educación y formación de audiencias para la apreciación estética.

- Fomentar la documentación, difusión y publicación de los proyectos realizados. Medialabcuenca.org (2016). 
Sus equipamientos, con los cuales consiguen perpetrar proyectos como Mapa sonoro de Cuenca (2016), En el lugar más lejano del mundo (2015) o Interfaces de Diálogo (2013) y un gran número de talleres, son adquiridos gracias a la financiación directa del Decanato de la Facultad de Artes, contando con buenos y actuales sistemas de iluminación profesional, equipo fotográfico y audiovisual o plotters e impresoras. Destaca por encima de todo la apuesta realizada por los kits electrónicos y sensores, tecnologías que se encuentran en la orilla del acceso abierto y el software libre, hecho que les permite mantener cierta coherencia con las prácticas y los procesos implementados en un alto porcentaje de los medialabs internacionales:

Cualquier centro cultural o laboratorio que pretenda actualizarse e implementar procesos culturales participativos vinculados al modelo de la read \& write culture, además de desarrollar organizaciones transdisciplinares, deberá implementar y promulgar el uso y desarrollo de recursos abiertos, pues son los únicos que permiten generar participación de la comunidad sobre los mismos (Ruiz y Alcalá, 2016, p. 235).

Cabe señalar que el MediaLab de la Facultad de Artes de la Universidad de Cuenca se plantea como un espacio de encuentro para estudiantes, artistas, docentes y diseñadores. En su plan de acción queda reflejado el desarrollo de proyectos interdisciplinares de arte y nuevas tecnologías desde una perspectiva abierta, con el fin de facilitar el conocimiento libre y la transferencia del mismo.

Por su parte, la Universidad Central del Ecuador, a través de su Facultad de Artes y gracias a la iniciativa de docentes e investigadores de la misma (que se comienza a generar en el contexto del Centro de Arte Contemporáneo de Quito), se plantea la necesidad de generar un espacio de encuentro y producción creativa. Este debe configurar transversalmente una comunidad formada por alumnos, docentes e investigadores y colaboradores de la Universidad interesados en impulsar proyectos que doten de contenido al laboratorio.

Debido a las carencias de las mallas curriculares señaladas, el proyecto UCE Lab, que actualmente se encuentra en su primera fase fundacional, se propone como el impulso necesario para situarse en un lugar privilegiado en investigación artística con nuevos medios, auténtico talón de Aquiles de la enseñanza universitaria en el Ecuador.

Se plantean cuatro líneas estratégicas fundamentales:

- Acción social y ciudadanía. Con especial atención a la participación ciudadana, al urbanismo y la arquitectura, a la ecología y el reciclaje, a la conciencia de género o al copyleft y Creative Commons. 
- Laboratorio de Turismo. Mediación turística, gastronomía, free-tours, etc.

- Laboratorio de Arte Multimedia. Sonido, audiovisual, gráfica digital 2D y 3D, interactivos, animación, cine, teatro y diseño.

- Makespace. Fabricación y prototipado digital: impresión 3D, tecnología CNC, Arduino, etc.

Tabla 2: Prácticas implementadas en los MEDialabs

ANALIZADOS SEGÚN OBJETIVOS /LÍNEAS ESTRATÉGICAS

\begin{tabular}{|c|c|c|}
\hline Indicadores & $\begin{array}{l}\text { Facultad de Artes } \\
\text { Universidad Central del Ecuador }\end{array}$ & $\begin{array}{l}\text { Facultad de Artes } \\
\text { Universidad de Cuenca }\end{array}$ \\
\hline \multicolumn{3}{|l|}{ Técnicas tradicionales } \\
\hline $\begin{array}{l}\text { Historia, teoría y } \\
\text { estudios del arte }\end{array}$ & $\begin{array}{l}\text { Conferencias abiertas de reflexión y } \\
\text { diálogo en torno a los nuevos } \\
\text { paradigmas del arte }\end{array}$ & $\begin{array}{l}\text { Conferencias abiertas de } \\
\text { reflexión y diálogo en torno a } \\
\text { los nuevos paradigmas del arte }\end{array}$ \\
\hline Gestión cultural & Laboratorio de Turismo & \\
\hline Investigación & $\begin{array}{l}\text { Propuesta de proyectos de investigación } \\
\text { transversales y vinculados con la } \\
\text { comunidad. }\end{array}$ & $\begin{array}{l}\text { Desarrollo de proyectos de } \\
\text { investigación transversales y } \\
\text { vinculados con la comunidad. }\end{array}$ \\
\hline Nuevas tecnologías & $\begin{array}{l}\text { Laboratorio de Arte Multimedia y } \\
\text { Makespace. Uso de nuevos medios } \\
\text { herramientas de fabricación digital } \\
\text { abiertas (open sources) }\end{array}$ & $\begin{array}{l}\text { Uso continuo de nuevas } \\
\text { herramientas de fabricación } \\
\text { digital abiertas (open sources) }\end{array}$ \\
\hline Otros datos & $\begin{array}{l}\text { Espacio de Acción social y ciudadanía. } \\
\text { Inclusión de otras carreras en los } \\
\text { procesos creativos. }\end{array}$ & $\begin{array}{l}\text { Formación continua mediante } \\
\text { talleres multidisciplinares. } \\
\text { Inclusión de otras carreras en } \\
\text { los procesos creativos. }\end{array}$ \\
\hline
\end{tabular}

Fuente: Elaboración propia basándonos en los materiales recabados.

Sus principales objetivos se centran en desarrollar proyectos de investigación y formación destinados a crear conciencia social y participación ciudadana en torno a temas de interés comunitario. A mejorar sustentablemente la calidad de la oferta turística de la ciudad, aplicando enfoques y perspectivas creativas. A generar proyectos artísticos en los que el pensamiento, la innovación, los nuevos medios, la creación colectiva y la fabricación digital sean los procesos que den respuesta a los paradigmas actuales. Esto nos aporta información 
muy relevante sobre el amplio campo de trabajo de dicho proyecto, aún no implementado, pues como se apuntó con anterioridad, se encuentra en pleno proceso fundacional. De todos modos, se espera que UCE Lab se convierta en el espacio de encuentro, diálogo, reflexión y desarrollo de proyectos y estudios en base al arte, la ciencia y la tecnología más importante del país, diferenciándose del anterior, principalmente, en sus amplias líneas de trabajo que abarcan desde la fabricación digital hasta el turismo o el urbanismo del siglo 21. Se trata de implementar el uso de los nuevos medios en todos sus procesos y difundir concienciación en relación al conocimiento gratuito y de libre acceso.

Así, las características, líneas y objetivos planteados por estos dos centros encajan con las políticas creativas seguidas por los medialabs actuales, aquellos que se integran en los cuatro ejes de la cultura participativa actual ${ }^{5}$, como lo son Medialab Prado (Madrid), eTopía (Zaragoza) o CCCBLAB (Barcelona) -en el caso nacional-, o Constant Variable (Bruselas) -a nivel internacional-, entre otros.

En estos medialabs, como norma general, no se priorizan espacios físicos dedicados a los artistas para que produzcan sus proyectos de forma individual, como los primeros centros. Estos espacios (...) se convierten en zonas amplias de intercambio de conocimientos, en espacios colectivizados donde incubar los proyectos. Funcionan más como un nodo, como catalizador y dinamizador de comunidades (Ruiz, 2014, p. 304).

En este sentido, actualmente existen dos aspectos principales: el papel de los públicos y la organización de los saberes en áreas de conocimiento. En relación a esto último, es una realidad insoslayable que la cultura digital emergente pone en tela de juicio dicha fragmentación. Quizás una de las cosas más interesantes de la cultura digital más actual es la conexión integradora de los saberes y del conocimiento. El concepto de experto ha variado radicalmente. Por eso se hacen necesarios espacios físicos donde los saberes se puedan conectar, sin jerarquías, que evite muchos de los planteamientos que históricamente se han desarrollado en el terreno artístico.

Nos importan los proyectos. Al fin y al cabo son los que articulan la construcción de co munidad, y esta es una de las claves de la cultura. La gente se reúne en torno a ideas concretas propuestas por cualquier individuo para desarrollar proyectos $\mathrm{y}$, a partir de estos proyectos, se construye una comunidad de personas que desarrollan sus capacidades en los

${ }^{5}$ Read \& write culture como modelo cultural participativo, transdisciplinariedad como modelo organizativo, open \& free sources como medios creativos y copyleft como método expansivo (Ruiz \& Alcalá, 2016). 
mismos. Las contribuciones y los resultados de cada proyecto suelen ofrecerse con licencias abiertas, lo que permite que otros puedan trasladarlos a otros lugares, es decir, pasan a formar parte del dominio público (García, comunicación personal, 2013).

\section{Conclusiones}

Tras analizar los materiales y exponer las características tanto de las mallas curriculares de las facultades como de las líneas estratégicas de los medialabs, cabe concluir que:

- El medialab de la universidad pública ecuatoriana viene a complementar en gran medida a las mallas curriculares y prácticas educativas desarrolladas en las facultades artísticas de las respectivas universidades. Esto se lleva a cabo mediante la actualización de conocimientos y la implementación de prácticas comunicativas y culturales actuales, ligadas a la cultura digital, a la contemporaneidad y a los cambios de paradigmas que las nuevas tecnologías ocasionan en materia educativa, creativa y cultural.

- Estos centros significan las primeras experiencias verdaderamente relevantes en cuanto a la convergencia del arte, la ciencia y la tecnología en Ecuador. Lo que es aún más importante, están impulsados por el sector público universitario, facilitando la integración y fomentando la participación de la sociedad en sus procesos, lo que generará en unos años -y valga como hipótesis- un importante cambio en la percepción y el imaginario colectivo del arte en el país.

- Sus objetivos, roles específicos y características relacionadas con los ejes de la cultura participativa actual son:

$>$ Generar espacios culturales, creativos, educativos y colaborativos de encuentro, donde se genere conocimiento y debate entre docentes, investigadores, alumnos y ciudadanos mediante la implementación de un modelo organizativo transdisciplinar. Read \& write culture como modelo cultural participativo.

$>$ Desarrollar proyectos de investigación transversales y con una marcada vinculación con la comunidad. Transdisciplinariedad como modelo organizativo.

$>$ Promover y facilitar recursos tecnológicos y documentales a la comunidad universitaria y no universitaria. Open \& free sources como medios creativos

$>$ Difundir y formar en el uso de los nuevos medios tecnológicos de licencias abiertas y acceso gratuito. Copyleft como método expansivo. 
- Los dos centros estudiados implementan en buena medida las nuevas prácticas culturales puestas en práctica en los medialabs más actuales, los mismos que beben de procesos dados en ambientes virtuales y fomentados por la nueva cultura digital. Así pues, resulta de gran relevancia que, en un país donde ha existido una ausencia notable de estudio y práctica del media art, las primeras experiencias se esfuercen por cubrir las expectativas de la nueva cultura mencionada. Esto indica un gran esfuerzo por parte de las instituciones de educación pública superior del país por actualizar sus contenidos de forma indirecta mediante proyectos satélites como son, en este caso, los medialabs ecuatorianos aquí estudiados. 


\section{Referencias}

Alcalá, J. R. \& Maisons, S. (2004). Estudio/Propuesta para la creación de un Centro de Excelencia en Arte y Nuevas Tecnologías. Madrid: Fundación Telefónica.

Alsina, P. (2007). Arte, ciencia y tecnología. Barcelona: EdiUOC.

Anderson, C. (2012). Makers. The New Industrial Revolution. New York: Crown Business.

Carlón, M. \& Scolari, C. A. (comps.) 2012. Colabor_arte. Medios y artes en la era de la producción colaborativa. Buenos Aires: La Crujía.

Century, M. (1999). Pathways to Innovation in Digital Culture. Centre for Research on Canadian Cultural Industries and Institutions / Next Century Consultants. Recuperado de $<$ http://www.nextcentury.ca/PI/PI.html $>$

De Vicente, J. L. (2008, 29 de mayo). Pioneros del 'media lab'. Robert Rauschenberg, entre arte y tecnología. El Cultural. Recuperado de $<$ http://www.elcultural.es/articulo_imp.aspx?id=23282>

García, M. Comunicación personal, Medialab Prado, 23 de septiembre de 2013.

(García, comunicación personal, 23 de septiembre de 2013).

García Carrizo, J. y Heredero Díaz, O. (2015). Propuesta de un modelo genérico de análisis de la estructura de las narrativas transmedia, Icono 14, volumen (13), pp. 260-285. $<$ doi: 10.7195/ri14. v13i2.745>

Hertz, G. (1995). The Godfather of Technology and Art: An interview with Billy Klüver. Conceptlab. Recuperado de $<$ http://www.conceptlab.com/interviews/kluver.html $>$

Jenkins, H. (2006). Convergence Culture: Where Old and New Media Collide. Nueva York: New York University Press.

Knobel, M. \& LANKshear, C. (2011). Remix: la nueva cultura popular. Cuadernos Comillas, 1, pp. $105-126$.

Lessig, L. (2005a). Por una cultura libre. Cómo los grandes grupos de comunicación utilizan la tecnología y la ley para clausurar la cultura y controlar la creatividad. Trad. A. Córdoba/elástico.net. Madrid: Traficantes de sueños.

Lessig, L. (2005b). Re:MixMe. Plenary address to the annual Network for IT-Research and Competence in Education (ITU) Conference, Oslo, Noruega. Octubre. 
Lessig, L. (2008). Remix: Making Art and Commerce Thrive in the Hybrid Economy. Nueva York: The Penguin Press.

Martín García, T. y Salóm Marco, E. (2013): Los colectivos artísticos: microcosmos y motor del procomún de las artes. Teknokultura, volumen (10), nº 1, pp. 49-74.

Martín Prada, J. (2012). Otro tiempo para el arte. Cuestiones y comentarios sobre el arte actual. Valencia: Sendemá.

McLuhan, M. (2009). Comprender los medios de comunicación. Las extensiones del ser humano. Trad. P. Ducher. Barcelona: Paidós.

Medialabucuenca.org, (2016). Historia y Objetivos - MediaLab. [En línea] Disponible en: $<$ http://www.medialabucuenca.org/acerca-de/sobre-el-laboratorio/historia-y-objetivos/> [Acceso 31 Ene. 2016].

Ruiz Martín, J.M. (2016): El crecimiento de los open sources de fabricación digital y su implementación en el media lab. De la high-tech al do it yourself, Comhumanitas: Revista científica de comunicación, 6-1, 67-81. ISSN: 1390-776X.

Ruiz Martín, J.M. y Alcalá Mellado, J.R. (2016): Los cuatro ejes de la cultura participativa actual. De las plataformas virtuales al medialab, Icono14, 14 (1), pp. 215-237. http://www.icono14.net/ojs/index.php/icono14/article/view/904 doi: 10.7195/ri14.v14i1.904

Ruiz Martín, J. M. (2016). De la producción artística de élite a la museografía. Estudio de caso: ZKM, Arte y Sociedad, volumen (10), 95-122. ISSN: 2174-7563

Ruiz Martín, J. M. (2014). Aparición, impacto y efectos de la máquina automática en el atelier del artista. Del taller tradicional al medialab. (Tesis inédita de doctorado). Cuenca: Departamento de Arte, Facultad de Bellas Artes de Cuenca, UCLM.

Ruiz Martín, J. M. (2011). El laboratorio actual de Gráfica Digital. Checking \& testing de software y hardware de alto rendimiento con fines creativos. Cuenca: MIDECIANT Graph.

Scolari, C. A. (2008). Hipermediaciones. Elementos para una teoría de la comunicación digital interactiva. Barcelona: Gedisa.

Wardrip-Fruin, N. \& Montfort, N. (2003). The New Media Reader. Massachusetts: The MIT Press. 